\title{
Soft Finger Model with Adaptive Contact Geometry for Grasping and Manipulation Tasks *
}

\author{
Matei Ciocarlie Claire Lackner Peter Allen \\ Columbia University, New York, USA \\ E-mail: cmatei@cs.columbia.edu,cnl2007@columbia.edu, allen@cs.columbia.edu
}

\begin{abstract}
This paper presents a method for building analytical contact models for soft fingers. Friction constraints are derived based on general expressions for non-planar contacts of elastic bodies, taking into account the local geometry and structure of the objects in contact. These constraints are then formulated as a linear complementarity problem, the solution of which provides the normal and frictional forces applied at each contact, as well as the relative velocity of the bodies involved. This approach captures frictional effects such as coupling between tangential force and frictional torque. We illustrate this method by analyzing manipulation tasks performed by an anthropomorphic robotic hand equipped with soft fingerpads.
\end{abstract}

\section{Introduction}

As dynamic simulation systems become more accurate at modeling the real world, the number of possible applications for such systems increases. One example is the dynamic simulation of grasping and manipulation tasks, enabling interaction with virtual objects. However, accurate grasping simulation requires modeling subtle physical phenomena not taken into account by traditional multi-rigidbody dynamic systems.

The ability to create stable, encompassing grasps with subsets of fingers is greatly increased by using soft fingertips that deform during contact and apply a larger space of frictional forces and moments than their rigid counterparts. This is true not only for human grasping, but also for robotic hands using fingerpads made of soft materials.

In this paper, we extend our previous research on grasp simulation $[12,13]$ to account for the complex nature of soft finger contacts. Interactive simulation rates are achieved using an analytical contact model based on the linear comple-

* This work is supported by the National Science Foundation Grant No. IIS-03-12693 and the Columbia College Rabi Scholars Program. mentarity formulation presented in [1]. In section 3 we provide an overview of this method focusing on those aspects relevant to frictional soft finger contacts.

Current analytical models for soft finger contacts make strong assumptions regarding the geometry of the objects in contact. The fingerpad is often modeled as a hemisphere, while the grasped object is assumed to be locally planar. We propose using more general contact models that can account for non-planar objects in contact (section 4) and discuss the integration of these models within the linear complementarity framework. In section 5 we show how this method can improve simulation accuracy using examples of an anthropomorphic hand performing manipulation tasks. Finally, we present our conclusions and plans for future research.

\section{Related Work}

One of the most accurate ways of simulating complex and irregular geometry or layered structures in contact is Finite Element Analysis. While this method has been used for the analysis of soft fingertips [17, 4], high computational requirements make it difficult to use for interactive dynamic simulations.

Computational performance suited for haptic rendering can be achieved by using analytical models for soft finger frictional contacts. We refer the reader to [2] for a comparison of 4 such models of the human fingertip, with different rotational friction properties. This work is extended in [7] to account for coupled frictional force and moments using the limit surface concept $[8,10]$ that our work also relies on. Soft finger models are then used for haptic interaction using an extension of the god-object algorithm [18].

A haptic rendering algorithm for frictionless deformable/rigid contacts using a linear complementarity formulation for contact constraints is presented in [6]. In [15], the authors present an optimized finite-element based method for real-time simulation of pinch grasps using deformable fingers. This method is shown to be highly efficient from a computational point of view, however it is cur- 


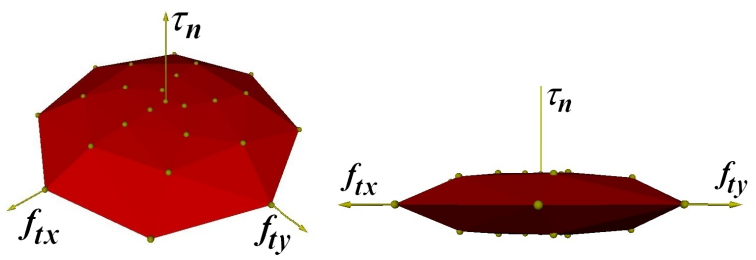

Figure 1. Friction ellipsoid with $e_{n}=0.2$

rently applied for two-dimensional cases using a Coulomb friction model. The problem of controlling a robotic finger when the dynamic properties of the deformable fingerpad are unknown is discussed in [9], and the control of soft finger rolling manipulation in [5].

\section{Linear complementarity formulation for contact problems with friction}

Consider a contact between two bodies, with a total load of magnitude $P$ applied in the direction of the contact normal $\hat{\boldsymbol{n}}$. In the case of rigid bodies creating a point contact, the Coulomb friction model states that frictional force can be applied in the tangent plane of the contact, and its magnitude $f_{t}$ is restricted by the load at the contact as well as the friction coefficient $\mu: f_{t} \leq \mu P$. In the case of soft fingers, the contact occurs over some area that increases as the normal force increases. As a result, it is also possible to apply a frictional moment of magnitude $\tau_{n}$ about the contact normal.

In order to express the constraint relating the magnitudes of frictional force and moment, we will use the results presented in [10]. An analytical expression for this relation depends on the pressure distribution inside the contact, and can only be derived for a limited number of special cases. However, it is shown that for the general case we can use an approximation of the following form:

$$
f_{t}^{2}+\frac{\tau_{n}^{2}}{e_{n}^{2}} \leq \mu^{2} P^{2}
$$

This equation describes what we will call the friction ellipsoid: by plotting the components of the frictional force along two perpendicular axes in the contact tangent plane against the magnitude of the frictional moment, the result is an ellipsoid of height $e_{n}$ (Fig. 1). We will refer to $e_{n}$ as the eccentricity parameter, which is set depending on the material properties and structure of the objects in contact. Its contribution to the contact model will be discussed in detail in section 4

Considering a friction wrench $\boldsymbol{f}_{w}$ combining both frictional force and torque, we can now intuitively express contact friction constraints. Any possible friction wrench applied at the contact has to be inside the friction ellipsoid and its force and torque components must satisfy eq. (1). If the friction wrench is strictly interior to the ellipsoid then no slip can occur at the contact. If slip does occur, then the frictional wrench must lie on the surface of the friction ellipsoid, and the directions of its force and torque components will oppose those of relative contact motion.

In practice, we use a linearized version of the ellipsoid obtained by taking the convex hull of a number $u$ of vertices on its surface. Instead of using eq. (1), we constrain the friction wrench to lie inside the discrete ellipsoid by formulating it as a linear combination of the vectors defined by the sampled vertices, with additional constraints on the chosen weights:

$$
\boldsymbol{f}_{w}=\boldsymbol{D} \boldsymbol{\beta} \mid \boldsymbol{\beta} \geq 0, e^{T} \boldsymbol{\beta} \leq \mu P
$$

where $e=[1,1, \ldots, 1]^{T} \in \mathcal{R}^{u}$, the columns of $\boldsymbol{D}$ contain the vectors that sample the friction ellipsoid and $\boldsymbol{\beta} \in \mathcal{R}^{u}$ is a vector of weights showing the contribution of each of these vectors to the resulting friction wrench.

Taking into account this formulation, we can express contact friction constraints as two complementarity conditions:

$$
\begin{aligned}
e \lambda+D^{T} \boldsymbol{v} & \geq 0 \text { compl. to } \boldsymbol{\beta} \geq 0 \\
\mu P-e^{T} \boldsymbol{\beta} & \geq 0 \text { compl. to } \lambda \geq 0
\end{aligned}
$$

where the vector $\boldsymbol{v}$ contains the relative velocities of the bodies involved and the additional variable $\lambda$ is in most cases an approximation of the magnitude of slip at the contact [1].

A complete contact model also requires that the normal force can only be compressive $(P \geq 0)$ and prevent interpenetration $\left(\hat{\boldsymbol{n}}^{T} \boldsymbol{v} \geq 0\right)$. These constraints are complementary (contact forces can only be applied as long as the bodies remain in contact), and can be summarized as follows:

$$
\hat{\boldsymbol{n}}^{T} \boldsymbol{v} \geq 0 \text { compl. to } P \geq 0
$$

Consider a complex simulation world, containing one or more virtual hands, graspable objects as well as static obstacles (such as floors or walls). For each contact that is created (regardless of whether it involves a finger, object or obstacle), linear complementarity constraints (3), (4) and (5) can be set up and assembled in matrix form. The resulting Linear Complementarity Problem (LCP) is solved using Lemke's algorithm, providing not only the normal and frictional forces applied at each of the contact points, but also the new velocities of the bodies [14]. We can then use a numerical integration scheme to compute the motion of each dynamic body in the simulation world. This framework therefore enables the study of virtual object interaction with a hand model as well as other virtual objects or obstacles, by using a unified treatment for all occurring contacts. In section 5 we will exemplify by showing manipulation results 
involving a hand with soft fingerpads and an object that is partially supported by a rigid table surface.

\section{Adaptive contact models}

We have mentioned in the previous section that the eccentricity parameter $e_{n}$ of the friction ellipsoid is set depending on the characteristics of the objects in contact. Following eq. (1), this parameter captures the relationship between maximum frictional force and moment that can be applied at the contact:

$$
e_{n}=\frac{\max \left(\tau_{n}\right)}{\max \left(f_{t}\right)}
$$

For a given magnitude $P$ of the contact normal force, the value of maximum tangential friction $\max \left(f_{t}\right)$ can easily be computed as $P \mu$. The value of the maximum frictional moment however depends on more complex factors, such as contact geometry and pressure distribution. In this section we describe a method that takes these factors into account and computes the value of the eccentricity parameter depending on the characteristics of the contact.

\subsection{Relative radii of curvature for non- planar contacts}

Using the formulation of [11], consider a local contact coordinate system with the origin at the center of the contact and the $z$ axis aligned with the contact normal. For two contacting bodies identified by the subscript $i$, we locally approximate their surfaces using an expression of the form

$$
z_{i}=A_{i} x^{2}+B_{i} y^{2}+C_{i} x y, \quad i \in\{1,2\}
$$

making the assumptions that the objects are locally smooth. The separation $h$ between the two surfaces is $z_{1}-z_{2}=$ $\left(A_{1}-A_{2}\right) x^{2}+\left(B_{1}-B_{2}\right) y^{2}+\left(C_{1}-C_{2}\right) x y$. By choosing the orientation of the $x$ and $y$ axes so that the term in $x y$ vanishes, the resulting equation may be re-written as:

$$
h=\frac{1}{2 R^{\prime}} x^{2}+\frac{1}{2 R^{\prime \prime}} y^{2}
$$

where $R^{\prime}$ and $R^{\prime \prime}$ are the relative radii of curvature of the objects in contact, depending only on their local geometry.

Our simulation environment considers objects as threedimensional meshes, which enables the use of numerous existing triangle mesh models, as well as efficient collision detection algorithms. When initial contact between two objects is detected, we approximate the shape of each object in a small region around the contact, using an analytical surface as described above. Since the resulting surface is expected to fit the original mesh only in a small area close to

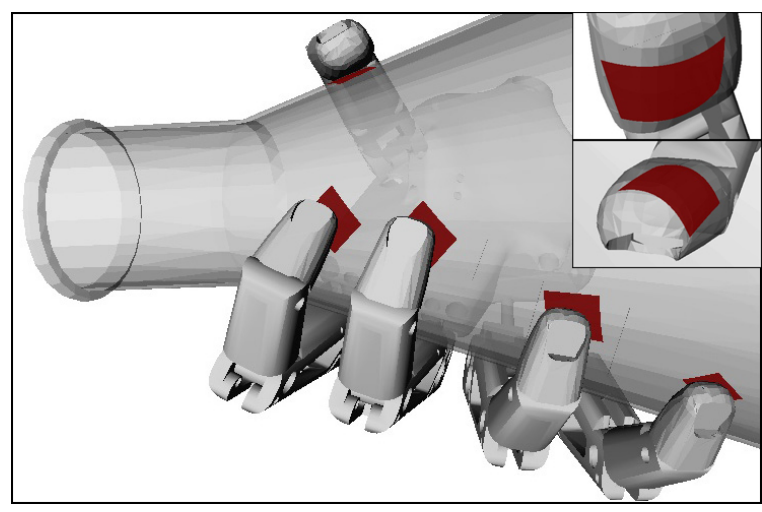

Figure 2. Analytical surfaces approximating the local geometry of a grasped object. Magnifications show local approximations for the thumb and little finger.

the contact region, we can obtain very close approximations using the relatively simple surface form of eq. (7).

Figure 2 exemplifies the result of our fitting method on robot fingers as well as the grasped object. We have used the fingertip model of the Shadow Robot anthropomorphic hand, currently in commercial production [16]. The quality of the approximation was computed as the standard deviation of the distance between the fitted surface and the original mesh, measured over a local region twice as large as the contact area predicted using the methods from section 4.2. Considering all the fitting results shown in figure 2, the largest value was reported for the thumb contact, with a standard deviation of $0.94 \mathrm{~mm}$ over an area of $82 \mathrm{~mm}^{2}$.

\subsection{Pressure distribution models}

Having computed the relative radii of curvature $R^{\prime}$ and $R^{\prime \prime}$, we can express the pressure distribution inside the contact using non-planar models that take into account the local geometry of the objects involved. We have performed this analysis for two pressure distribution models. The first is the Hertzian model, previously used in the literature to simulate both human and robotic soft fingertips $[2,3]$. The second is the Winkler elastic foundation model, which can be used in the case of an elastic layer resting on a rigid base, such as a robotic fingertip coated in a thin layer of soft material.

For both of these models, the contact area is known to be elliptical in shape [11], with semi-axes $a$ and $b$ depending, among other factors, on the relative radii of curvature at the contact. In the case of the Winkler foundation model, the ratio of frictional torque to contact load can be computed 
as:

$$
\frac{\max \left(\tau_{n}\right)}{P}=\frac{8}{15} \mu \sqrt{a b}
$$

Alternatively, in the case of a Hertzian pressure model the relationship is:

$$
\frac{\max \left(\tau_{n}\right)}{P}=\frac{3 \pi}{16} \mu \sqrt{a b}
$$

Due to space constraints, we are unable to include full derivations for eqs. (9) and (10), however a brief description in the case of the Winkler foundation model is given in Appendix A. The case of the Hertzian model can be handled in a similar manner, based on the results of $[11,2]$. It is important to note that this method can also be applied for other pressure distribution models. If the value of the maximum frictional moment can be computed using the chosen model together with the local geometry of the colliding objects, then the contact friction ellipsoid can be built using the methods described below.

We can now summarize the algorithm used for setting up soft contacts. Starting when initial contact between two bodies is detected, we perform the following steps:

- use a least squares method to fit a surface of the form of eq. (7) to each of the bodies involved

- compute the relative radii of curvature at the contact

- choose a model for the pressure distribution inside the contact that best fits the objects involved

- use the pressure distribution model and the relative radii of curvature to compute the dependency between contact normal force and maximum frictional torque

- compute the eccentricity parameter of the friction ellipsoid using eq. (6) and set up linear complementarity conditions (3) - (5) for the contact

\subsection{Discussion}

The analytical pressure distribution models that we have discussed (the Hertzian model and the Winkler foundation) share a number of assumptions regarding the bodies in contact. These assumptions include a linear relationship between stress and strain, homogeneous inner structure of the objects, and small deformations due to contact (relative to the total size of the objects). Such assumptions do not always hold true in the case of the human finger, and computationally intensive methods such as Finite Element Analysis are needed in order to fully account for such phenomena. However, it has been shown [2] that fast analytical models can provide close approximations when characterizing soft finger contacts. The proposed algorithm maintains the computational advantages associated with an analytical contact model, while taking into account variations in fingertip and object geometry.
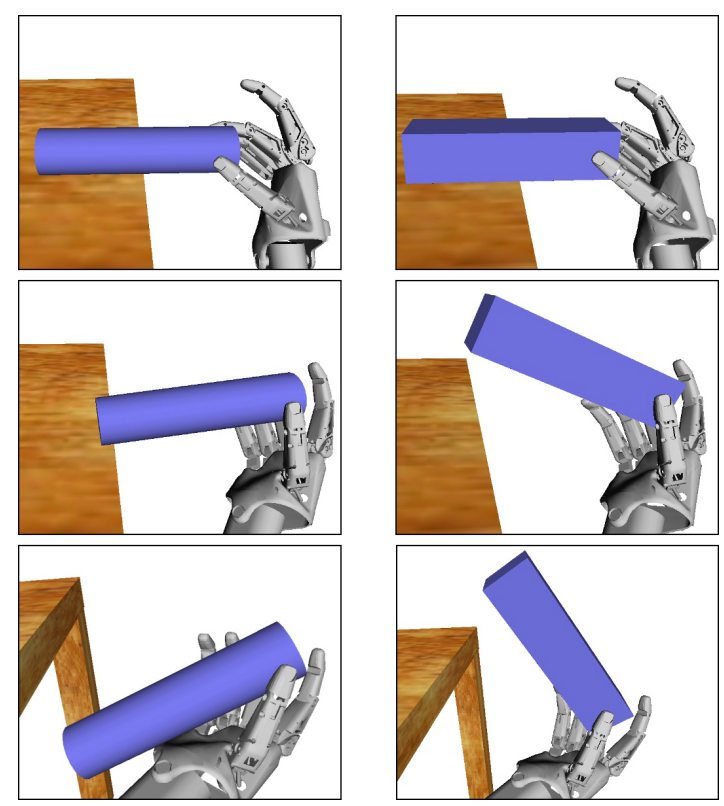

Figure 3. Example of relative radii of curvature affecting the magnitude of the frictional torque supported by the contacts.

To exemplify, we will again consider the fingertip contacts shown in Fig. 2. For an identical load of $5 \mathrm{~N}$ at each contact, we have used the elastic foundation model to compute the contact area created by each finger as well as the maximum value of contact frictional torque. The obtained results for the contact area varied between $41 \mathrm{~mm}^{2}$ for the thumb and $26 \mathrm{~mm}^{2}$ for the ring finger. As a result, frictional torque applied at the thumb contact can be approximately $30 \%$ higher than the torque applied at the ring finger. In the range of forces and moments required for common manipulation tasks, these variations can introduce significant differences. Simulation accuracy can be improved by considering their effects, as shown in the following section.

\section{Examples of simulated manipulation tasks}

We will first consider a case in which local curvature at the contact locations makes a significant difference on the final pose of a grasped object. Figure 3 shows a manipulation procedure used on two objects of similar material properties, with robotic fingers applying identical normal forces in both cases. In the case of a rectangular object, friction prevented any rotational slip at the contacts (figure 3, right column). However, in the case of an object displaying higher local curvature, smaller contact areas restricted the maximum magnitude of frictional torque. As a result, the fingers were unable to apply the necessary friction wrench 
in order to counter the effect of gravity: the object could not be lifted into a vertical position and was dragged across the table surface until falling into the palm (figure 3, left column).

An example of complex manipulation is presented in figure 4. The goal of the simulated task is to obtain a stable grasp of the glass-shaped object by using finger contacts around its circumference as well as contacts on the palm. However, in the initial position (figure 4, top left) the surface of the table prevents the execution of such a grasp. We have simulated a control algorithm that uses two fingers to pick up the object and rotate it above the palm (figure 4, top row). This is possible only in the presence of frictional torque applied by soft finger contacts. With the palm facing up (figure 4, bottom left), the force applied by the fingers is decreased allowing the glass to rotate while maintaining contact with the fingertips until it hits the rigid palm of the robot. The ring and little finger can now be closed around the glass, creating a stable grasp (figure 4, bottom right).

For the sequence shown in figure 4 , numerical integration was carried out at a rate of 10.7 time steps per second on a standard desktop computer, with the entire sequence consisting of 1800 time steps. At each time step, the most significant amount of computational effort was spent solving the system LCP and, in our current implementation, determining the motion of the robotic hand under the effect of joint motors and constraints.

We are currently considering two approaches for increasing computational efficiency. The first involves a human user providing the hand motion directly by using motion capture or haptic devices. The second aims to decrease the amount of time spent solving the system LCP by using the basis computed during the previous time step as a starting point for the current LCP [1]. Such methods can potentially target the necessary frame rate for haptic rendering of the fingertip forces (contact loads and friction) which are computed at each time step.

\section{Conclusions and future work}

In this paper we have presented a method for building an analytical model for frictional soft finger contacts based on the local geometry and structure of the bodies involved. General models for non-planar contacts of elastic bodies are used to derive the friction constraints that characterize the contact. These constraints are integrated into a linear complementarity formulation enabling fast dynamic simulation of multi-body interaction. We have used this method to simulate the behavior of robotic hands involved in manipulation tasks using both soft fingers and rigid body contacts.

Robotic hands are still a long way from matching the grasping and manipulation capability of their human counterparts, but computer simulation may help us understand this disparity. Our current efforts are focused on constructing a biomechanically realistic human hand model which would also allow us to determine which features are the most important to be mimicked when designing a robotic hand. This paper proposes a method for taking into account the space of forces that can be transmitted through a deformable fingerpad contact and its effects on the grasping ability of the hand. Iterative refinements of the model will include realistic human joints as well as indirect actuation applied through a network of tendons. Such a model would also serve to aid clinicians planning reconstructive surgeries of a hand, and creating more effective designs for hand prostheses.

\section{A Maximum frictional torque as a function of contact load}

Consider a Winkler elastic foundation, of depth $h$ and elastic modulus $K$, resting on a rigid base and in contact with a rigid object applying a total load $P$. Using the results of [11], the compression $\delta$ of the elastic layer caused by the indenter at the center of contact will be:

$$
\delta=\sqrt{\frac{P h}{K \pi\left(R^{\prime} R^{\prime \prime}\right)^{\frac{1}{2}}}}
$$

where $R^{\prime}$ and $R^{\prime \prime}$ are the relative radii of curvature computed as described in section 4.1. The contact area will be described by an ellipse of semi-axes $a=\sqrt{2 \delta R^{\prime}}$ and $b=\sqrt{2 \delta R^{\prime \prime}}$. The pressure distribution inside the contact area is:

$$
p(x, y)=\frac{K \delta}{h}\left(1-\frac{x^{2}}{a^{2}}-\frac{y^{2}}{b^{2}}\right)
$$

By integrating over the contact area we obtain the formula for total contact load:

$$
P=\frac{K \pi a b \delta}{2 h}
$$

According to [10], maximum frictional moment will be applied if relative contact motion is a rotation around the pressure-weighted center of the contact. The contribution of each contact point to the total friction moment is therefore

$$
m(x, y)=\sqrt{x^{2}+y^{2}} \mu p(x, y)
$$

By integrating eq. (14) over the entire contact area we obtain the value of the maximum frictional moment than can be applied at the contact

$$
\max \left(\tau_{n}\right)=\frac{K \delta}{h} \mu \frac{4 \pi}{15}(a b)^{\frac{3}{2}}
$$

and from (13) and (15) we obtain

$$
\frac{\max \left(\tau_{n}\right)}{P}=\frac{8 \mu}{15} \sqrt{a b}
$$



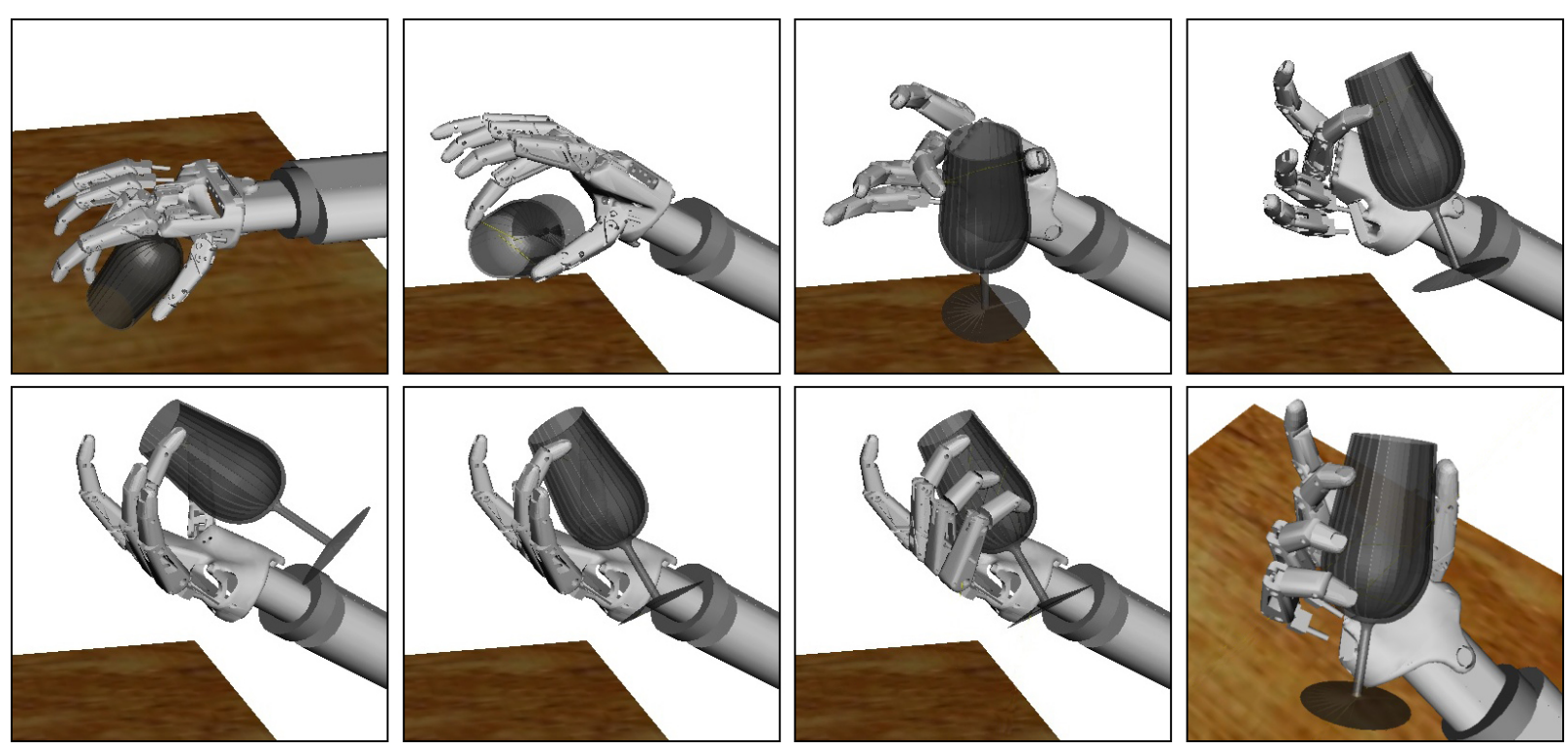

Figure 4. Simulation of a manipulation task using an anthropomorphic robotic hand. Top row: object is picked up and rotated above the palm. Bottom row: by controlling fingertip force, rotational sliding is allowed at the contacts until object rests on palm. By closing the fingers a stable grasp is obtained (bottom right). A complete movie showing this sequence is available at http://www.cs.columbia.edu/ cmatei/wh07

\section{References}

[1] M. Anitescu and F. A. Potra. Formulating dynamic multirigid-body contact problems with friction as solvable linear complementarity problems. Nonlinear Dynamics, 14:231247, 1997.

[2] F. Barbagli, A. Frisoli, K. Salisbury, and M. Bergamasco. Simulating human fingers: a soft finger proxy model and algorithm. In Intl. Symp. on Haptic Interfaces, pages 9-17, 2004.

[3] D. Brock. Enhancing the dexterity of a robot hand using controlled slip. In IEEE ICRA, pages 249-251, 1988.

[4] M. Ciocarlie, A. Miller, and P. Allen. Grasp analysis using deformable fingers. In IEEE Intl. Conf. on Intelligent Robots and Systems, pages 4122-4128, 2005.

[5] Z. Doulgeri and J. Fasoulas. Grasping control of rolling manipulations with deformable fingertips. IEEE/ASME Transactions on Mechatronics, 8(2):283-286, 2003.

[6] C. Duriez, C. Andriot, and A. Kheddar. A multi-threaded approach for deformable/rigid contacts with haptic feedback. In Intl. Symp. on Haptic Interfaces, pages 272-279, 2004.

[7] A. Frisoli, F. Barbagli, E. Ruffaldi, K. Salisbury, and M. Bergamasco. A limit-curve based soft finger god-object algorithm. In Intl. Symp. on Haptic Interfaces, pages 217223, 2006.

[8] S. Goyal, A. Ruina, and J. Papadopoulos. Planar sliding with dry friction, part 1. Wear, 143:307-330, 1991.

[9] H. Y. Han, S. Arimoto, K. Tahara, M. Yamaguchi, and P. Nguyen. Robotic pinching by means of a pair of soft fin- gers with sensory feedback. In IEEE Intl. Conf. on Robotics and Automation, pages 97-102, 2001.

[10] R. Howe and M. Cutkosky. Practical force-motion models for sliding manipulation. Intl. J. of Robotics Research, 15(6):557-572, December 1996.

[11] K. Johnson. Contact Mechanics. Cambridge University Press, 1985.

[12] A. Miller and P. K. Allen. Graspit!: A versatile simulator for robotic grasping. IEEE Robotics and Automation Magazine, 11(4):110-122, December 2004.

[13] A. Miller, P. K. Allen, V. Santos, and F. Valero-Cuevas. From robot hands to human hands: A visualization and simulation engine for grasping research. Industrial Robot, 32(1):55-63, 2005.

[14] A. Miller and H. Christensen. Implementation of multirigid-body dynamics within a robotic grasping simulator. In IEEE Intl. Conf. on Robotics and Automation, pages 22622268, 2003.

[15] M. Pouliquen, C. Duriez, C. Andriot, A. Bernard, L. Chodorge, and F. Gosselin. Real-time finite element finger pinch grasp simulation. In Joint Eurohaptics Conference and Symposium on Haptic Interfaces, pages 323-328, 2005.

[16] The Shadow Hand. http://www.shadowrobot.com/hand/.

[17] N. Xydas, M. Bhagavat, and I. Kao. Study of soft-finger contact mechanics using finite elements analysis and experiments. In IEEE Intl. Conf. on Robotics and Automation, pages 2179-2184, 2000.

[18] C. Zilles and J. Salisbury. A constraint-based god-object method for haptic display. In IEEE Intl. Conf. on Intelligent Robots and Systems, pages 146-151, 1995. 